\title{
Numerical Solutions of Volterra Integral Equations Using Laguerre Polynomials
}

\author{
M. A. Rahman ${ }^{1}$, M. S. Islam ${ }^{2 *}$ and M. M. Alam ${ }^{3}$ \\ ${ }^{1}$ Department of Mathematics and Statistics, Bangladesh University of Business and Technology, \\ Dhaka-1216, Bangladesh \\ ${ }^{2}$ Department of Mathematics, University of Dhaka, Dhaka-1000, Bangladesh \\ ${ }^{3}$ Mathematics Discipline, Khulna University, Khulna-9208, Bangladesh \\ Received 7 January 2012, accepted in final revised form 20 March 2012
}

\begin{abstract}
We solve numerically Volterra integral equations, of first and second kind with regular and singular kernels, by the well known Galerkin weighted residual method. For this, we derive a simple and efficient matrix formulation using Laguerre polynomials as trial functions. Several numerical examples are tested. The approximate solutions of some examples coincide with the exact solutions on using a very few Laguerre polynomials. The approximate results, obtained by the present method, confirm the convergence of numerical solutions and are compared with the existing methods available in the literature.
\end{abstract}

Keywords: Volterra integral equations; Galerkin method; Laguerre polynomials.

(c) 2012 JSR Publications. ISSN: 2070-0237 (Print); 2070-0245 (Online). All rights reserved.

doi: http://dx.doi.org/10.3329/jsr.v4i2.9407 J. Sci. Res. 4 (2), 357-364 (2012)

\section{Introduction}

In recent years, there has been a growing interest in the Volterra integral equations arising in various fields of physics and engineering [1], e.g., potential theory and Dirichlet problems, electrostatics, the particle transport problems of astrophysics and reactor theory, contact problems, diffusion problems, and heat transfer problems. Also many initial and boundary value problems associated with the ordinary and partial differential equations can be cast into the Volterra integral equations.

Some valid numerical methods, for solving Volterra equations using various polynomials [2], have been developed by many researchers. Very recently, Maleknejad et al. [3] and Mandal and Bhattacharya [4] used Bernstein polynomials in approximation techniques, Shahsavaran [5] solved by Block-Pulse functions and Taylor expansion

"Corresponding author: mdshafiqul@yahoo.com 
method. Taylor polynomials were also used by Bellour and Rawashdeh [6] and Wang [7] with computer algebra. Maleknejad et al. [8] studied first kind by using a Recursive scheme. Bernstein polynomials were used for the solution of second order linear and first order non-linear differential equations by Bhatti and Bracken [9]. These polynomials have also been used for solving Fredholm integral equations of second kind by Shirin and Islam [10].

However, in this paper a very simple and efficient Galerkin weighted residual [11] numerical method is proposed with Laguerre polynomials as trial functions. The formulation is derived to solve the linear Volterra integral equations of both first and second kind and/or having regular as well as weakly singular kernels, in details, in section 3. In section 2, we give a short introduction of Laguerre polynomials. Finally, five examples of different kinds of Volterra integral equations are given to verify the proposed formulation. The results of each example indicate the convergence numerical solutions. Moreover, this method can provide even the exact solutions, with a few lower order Laguerre polynomials, if the equation is simple.

\section{Laguerre Polynomials}

The general form of the Laguerre polynomials [2] of $n$th degree is defined by

$$
L_{n}(x)=\sum_{r=0}^{n}(-1)^{r} \frac{(n !)^{2}}{(n-r) !(r !)^{2}} x^{r}
$$

Using MATLAB code, the first few Laguerre polynomials from Eq. (1) are given below:

$$
\begin{aligned}
& L_{0}(x)=1 \quad L_{1}(x)=1-x \quad L_{2}(x)=\frac{1}{2}\left(x^{2}-4 x+2\right) \\
& L_{3}(x)=\frac{1}{6}\left(-x^{3}+9 x^{2}-18 x+6\right) \\
& L_{4}(x)=\frac{1}{24}\left(x^{4}-16 x^{3}+72 x^{2}-96 x+24\right) \\
& L_{5}(x)=\frac{1}{120}\left(-x^{5}+25 x^{4}-200 x^{3}+600 x^{2}-600 x+120\right) \\
& L_{6}(x)=\frac{1}{720}\left(x^{6}-35 x^{5}+450 x^{4}-2400 x^{3}+5400 x^{2}-4320 x+720\right)
\end{aligned}
$$

Now the first six Laguerre polynomials over the interval $[-5,20]$ are shown in Fig. 1. 


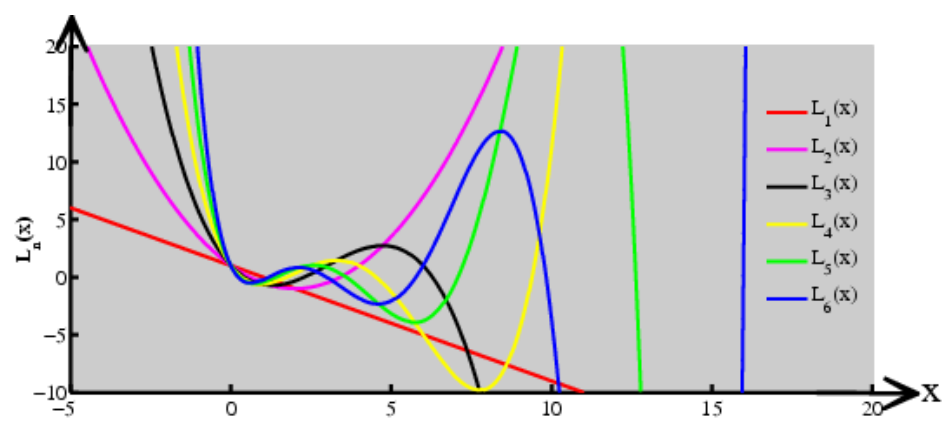

Fig. 1. Graph of first 6 Laguerre polynomials over the interval $(-5,20)$.

\section{Formulation of Integral Equation in Matrix Form}

The most standard form of Volterra linear integral equations (VIE) of the first kind [1] is of the form

$$
\int_{a}^{x} k(x, t) \varphi(t) d t=f(x), \quad a \leq x \leq b
$$

where $\varphi(x)$ is the unknown function, to be determined, $k(x, t)$ the kernel, is a continuous or discontinuous, $f(x)$ being the known function satisfying $f(a)=0$.

Now we use the Galerkin weighted residual method [11] to find an approximate solution $\tilde{\varphi}(x)$ of (2). For this, we assume that

$$
\tilde{\varphi}(x)=\sum_{i=0}^{n} a_{i} L_{i}(x)
$$

where $L_{i}(x)$ are Laguerre polynomials of degree $i$ defined in Eq. (1), $n$ is the number of Laguerre polynomials, and $a_{i}$ are unknown parameters, to be determined. Substituting (3) into (2), we get

$$
\sum_{i=0}^{n} a_{i} \int_{a}^{x} k(x, t) L_{i}(t) d t=f(x), a \leq x \leq b
$$

Then the Galerkin equations are obtained by multiplying both sides of (4) by $L_{j}(x)$ (as weight functions), and integrating the resulting equation with respect to $x$ over the interval $[a, b]$ to obtain

$$
\sum_{i=0}^{n} a_{i} \int_{a}^{b}\left[\int_{a}^{x} k(x, t) L_{i}(t) d t\right] L_{j}(x) d x=\int_{a}^{b} L_{j}(x) f(x) d x, j=0,1, \ldots \ldots, n
$$

Since in each equation, there are two integrals. The inner integrand of the left side is a function of $x$, and $t$ and is integrated with respect to $t$ from $a$ to $x$. As a result the outer integrand becomes a function of $x$ only and integration with respect to $x$ from $a$ to $b$ yields 
a constant. Thus for each $j=0,1, \ldots \ldots, n$ we have a linear equation with $n+1$ unknowns $a_{\mathrm{i}}, i=0,1, \ldots, n$.

Finally, Eq. (5) represents the system of $n+1$ linear equations in $n+1$ unknowns, are given by

$$
\sum_{i=0}^{n} a_{i} K_{i, j}=F_{j} ; i, j=0,1,2, \cdots \cdots, n
$$

where, $K_{i, j}=\int_{a}^{b}\left[\int_{a}^{x} k(x, t) L_{i}(t) d t\right] L_{j}(x) d x, i, j=0,1, \ldots \ldots, n$

$$
F_{j}=\int_{a}^{b} L_{j}(x) f(x) d x, \quad j=0,1, \ldots \ldots, n
$$

The unknown parameters $a_{i}$ are to be determined by solving the system of Eq. (6), and substituting these values of parameters in (3), we get the approximate solution $\tilde{\varphi}(x)$ of the integral Eq. (2).

Now, we consider the Volterra integral equation (VIE) of the second kind [1] given by

$$
c(x) \varphi(x)+\lambda \int_{a}^{x} k(x, t) \varphi(t) d t=f(x), \quad a \leq x \leq b
$$

where, $\varphi(x)$ is the unknown function to be determined, $k(x, t)$ the kernel, is a continuous or discontinuous. $f(x), c(x)$ are known functions and $\lambda$ is the constant. Then applying the same procedure described as above, we obtain

$$
\begin{aligned}
& \sum_{i=0}^{n} a_{i} K_{i, j}=F_{j} ; i, j=0,1,2, \cdots \cdots, n \\
& \text { where, } K_{i, j}=\left[\int_{a}^{b}\left[c(x) L_{i}(x)+\lambda \int_{a}^{x} k(x, t) L_{i}(t) d t\right] L_{j}(x) d x\right], i, j=0,1, \ldots \ldots, n \\
& F_{j}=\int_{a}^{b} L_{j}(x) f(x) d x, j=0,1, \ldots \ldots, n
\end{aligned}
$$

Here the unknown parameters $a_{i}$ are determined by solving the system of Eqs. (8) and substituting these values of parameters in (3), we get the approximate solution $\tilde{\varphi}(x)$ of the integral Eq. (7). The absolute error for this formulation is defined by

Absolute error $=|\varphi(x)-\tilde{\varphi}(x)|$

\section{Numerical Examples}

Here we illustrate the above mentioned methods with the help of five numerical examples, which include three first kind and two second kind with regular kernels and weakly singular kernels, available in the existing literature [1, 3- 5]. The computations, associated with the examples, are performed by MATLAB 7. The convergence of each linear Volterra integral equations is calculated by 


$$
E=\left|\tilde{\varphi}_{n+1}(x)-\tilde{\varphi}_{n}(x)\right| \prec \delta
$$

where $\tilde{\varphi}_{n}(x)$ denotes the approximate solution by the proposed method using $n$th degree polynomial approximation and $\delta$ varies from $10^{-6}$ for $n \geq 10$.

Example 1: Consider the VIE of first kind (page 154 [1])

$$
\int_{0}^{x} 3^{x-t} \varphi(t) d t=x, \quad 0 \leq x \leq 1
$$

The exact solution is $\varphi(x)=1-x \log _{e} 3$. Using the formula derived in the previous section and solving the system (6) for $n \geq 1$, we get the approximate solution is $\tilde{\varphi}(x)=1-x \log _{e} 3$, which is the exact solution.

Example 2: Consider an Abel's integral equation (VIE of first kind with weakly singular kernels) of the form [3]

$$
\int_{0}^{x} \frac{1}{\sqrt{(x-t)}} \varphi(t) d t=\frac{2}{105} \sqrt{x}\left(105-56 x^{2}+48 x^{3}\right) \quad 0 \leq x \leq 1
$$

The exact solution is $\varphi(x)=x^{3}-x^{2}+1$. Results have been shown in Table 1 for $n=10$. Also Fig. 2 shows the exact and approximate solutions on using 2, 3 and 11 Laguerre polynomials, respectively. The absolute errors obtain in the order of $10^{-16}$ on using Laguerre polynomials with $n=10$. On the other hand, the accuracy is found nearly the order of $10^{-7}$ by Maleknejad et al. [3] on using Bernstein approximation for $n=10$.

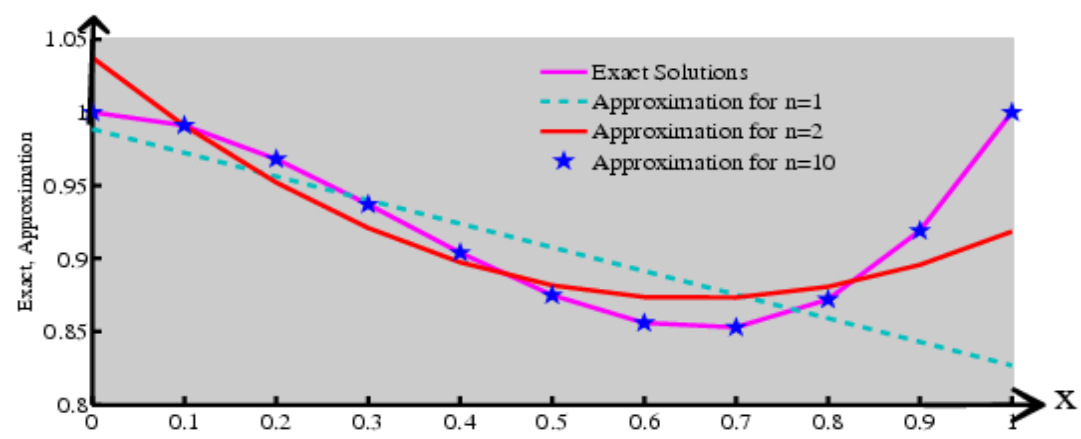

Fig. 2. Exact solutions and Numerical solutions of example 2 for $n=1,2$ and 10

Example 3: Consider an Abel's integral equation (VIE of first kind with weakly singular kernels) of the form [4] 
$\int_{0}^{x} \frac{1}{\sqrt{(x-t)}} \varphi(t) d t=x^{5}, 0 \leq x \leq 1$

The exact solution is $\varphi(x)=\frac{1280}{315 \pi} x^{9 / 2}$. Results have been shown in Table 1 for $n=10$. Also Fig. 3 shows the exact and approximate solutions on using 2, 3, 4 and 11 Laguerre polynomials, respectively. The absolute errors obtain in the order of $10^{-8}$ on using 11 Laguerre polynomials, while the accuracy were found in [4] by Bhattacharya and Mandal nearly the order of $10^{-7}$ on using 11 Bernstein polynomials.

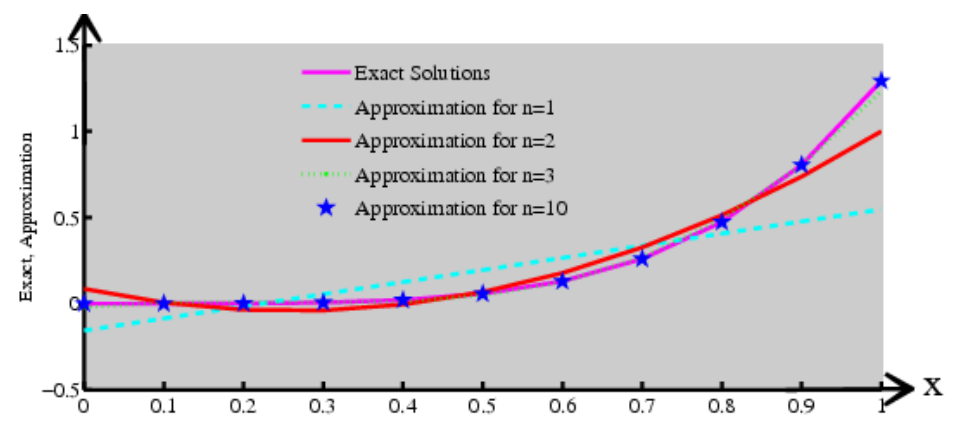

Fig. 3. Exact solution and numerical solution of example 3 for $n=1-3$ and 10.

Table 1. Computed absolute error of examples $2-4$ for $n=10$.

\begin{tabular}{llccccc}
\hline & \multicolumn{2}{c}{ Example 2 } & \multicolumn{2}{c}{ Example 3 } & \multicolumn{2}{c}{ Example 4 } \\
\cline { 2 - 7 } $\boldsymbol{x}$ & $\begin{array}{c}\text { Exact } \\
\text { Solutions }\end{array}$ & Absolute Error & $\begin{array}{c}\text { Exact } \\
\text { Solutions }\end{array}$ & $\begin{array}{c}\text { Absolute } \\
\text { Error }\end{array}$ & $\begin{array}{c}\text { Exact } \\
\text { Solutions }\end{array}$ & Absolute Error \\
\hline 0.0 & 1.0000000 & $0.0000000 \mathrm{E}+000$ & 0.0000000 & $0.0000000 \mathrm{E}+000$ & 0.0000000 & $0.0000000 \mathrm{E}+000$ \\
0.1 & 0.9910000 & $3.3306691 \mathrm{E}-016$ & 0.0000409 & $1.4502586 \mathrm{E}-008$ & 0.0000001 & $1.3790466 \mathrm{E}-011$ \\
0.2 & 0.9680000 & $0.0000000 \mathrm{E}+000$ & 0.0009255 & $7.8727334 \mathrm{E}-009$ & 0.0000128 & $1.5311828 \mathrm{E}-011$ \\
0.3 & 0.9370000 & $5.5511151 \mathrm{E}-016$ & 0.0057385 & $2.0173076 \mathrm{E}-008$ & 0.0002187 & $8.1805515 \mathrm{E}-012$ \\
0.4 & 0.9040000 & $7.7715612 \mathrm{E}-016$ & 0.0209421 & $8.4781877 \mathrm{E}-009$ & 0.0016384 & $2.4509244 \mathrm{E}-011$ \\
0.5 & 0.8750000 & $0.0000000 \mathrm{E}+000$ & 0.0571629 & $1.3312508 \mathrm{E}-008$ & 0.0078125 & $1.4551915 \mathrm{E}-011$ \\
0.6 & 0.8560000 & $1.6653345 \mathrm{E}-015$ & 0.1298465 & $2.4497113 \mathrm{E}-010$ & 0.0279936 & $4.6102080 \mathrm{E}-011$ \\
0.7 & 0.8530000 & $1.5543122 \mathrm{E}-015$ & 0.2598309 & $5.6635984 \mathrm{E}-008$ & 0.0823543 & $1.8066840 \mathrm{E}-010$ \\
0.8 & 0.8720000 & $1.1102230 \mathrm{E}-016$ & 0.4738648 & $3.6901042 \mathrm{E}-008$ & 0.2097152 & $5.3086729 \mathrm{E}-011$ \\
0.9 & 0.919000 & $4.4408921 \mathrm{E}-016$ & 0.8050833 & $2.0402750 \mathrm{E}-008$ & 0.4782969 & $8.3868856 \mathrm{E}-011$ \\
1.0 & 1.0000000 & $1.7763568 \mathrm{E}-015$ & 1.2934497 & $2.1811684 \mathrm{E}-007$ & 1.0000000 & $4.3655746 \mathrm{E}-011$ \\
\hline
\end{tabular}

Example 4: Consider the weakly singular VIE of second kind [4] 


$$
\varphi(x)-\int_{0}^{x} \frac{1}{\sqrt{(x-t)}} \varphi(t) d t=x^{7}\left(1-\frac{4096}{6435} \sqrt{x}\right), 0 \leq x \leq 1
$$

The exact solution is $\varphi(x)=x^{7}$. Results have been shown in Table 1 for $n=10$. Also Fig.4 shows the exact and approximate solutions on using 2, 3, 4 and 11 Laguerre polynomials, respectively. The absolute errors obtain in the order of $10^{-11}$ on using 11 Laguerre polynomials while the accuracy was found in Ref. [4] nearly the order of $10^{-7}$ using 11 Bernstein polynomials.

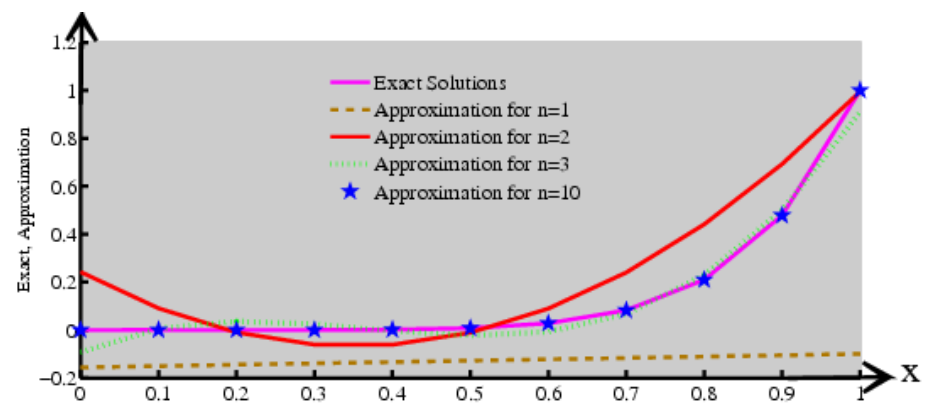

Fig. 4. Exact solutions and Numerical solutions of example 4 for $n=1-3$ and 10 .

Example 5: Consider the weakly singular VIE of second kind [5]

$$
\varphi(x)+\int_{0}^{x} \frac{1}{\sqrt{(x-t)}} \varphi(t) d t=x^{2}+\frac{16}{15} x^{\frac{5}{2}}, 0 \leq x \leq 1
$$

The exact solution is $\varphi(x)=x^{2}$. Using the formula derived in the previous section and solving the system (8) for $n \geq 2$, we get the approximate solution is $\tilde{\varphi}(x)=x^{2}$, which is the exact solution. On the contrary, the accuracy is found nearly the order of $10^{-3}$ for $n=32$ (number of Block-Pulse functions) by Shahsavaran [5] using Block-Pulse functions and Taylor expansion by collocation method.

\section{Conclusions}

A very simple and efficient Galerkin weighted residual method based on the Laguerre polynomial basis has been developed to solve first kind, second kind and also singular Volterra integral equations. Numerical results have been obtained in Table- 1 with great accuracy in comparison with the numerical results obtained by using Bernstein polynomials [3, 4] and also by other techniques. It is observed that the approximate results 


\section{Numerical Solutions}

converge monotonically to the exact solutions. We notice that the approximate solutions coincide with the exact solutions even a few of the polynomials are used in the approximation which are shown in examples 1 and 5 . We may realize that this method may be applied to solve other integral equations for the desired accuracy.

\section{References}

1. A. J. Jerri, Introduction to Integral Equations with Applications (John Wiley \& Sons Inc., New York, 1999).

2. N. Saran, S. D. Sharma, and T. N. Trivedi, Special Functions, $7^{\text {th }}$ edition (Pragati Prakashan, Meerut, India, 2000).

3. K. Maleknejad, E. Hashemizadeh, and R. Ezzati, Commun. Nonlinear Sci. Numer. Simulat. 16, 647 (2011). http://dx.doi.org/10.1016/j.cnsns.2010.05.006

4. S. Bhattacharya and B. N. Mandal, Appl. Math. Sci. 2, 1773 (2008).

5. A. Shahsavaran, Appl. Math. Sci. 5, 685 (2011).

6. A. Bellour and E. A. Rawashdeh, J. Inequal. Speci. Func. 1, 23 (2010).

7. W. Wang, Appl. Math. Comput. 172, 1323 (2006). http://dx.doi.org/10.1016/j.amc.2005.02.056

8. K. Maleknejad, M. Roodaki, and H. Almasieh, J. Math. Extens. 3, 113 (2009).

9. M. I. Bhatti and P. Bracken, J. Comput. Appl. Math. 205, 272 (2007). http://dx.doi.org/10.1016/j.cam.2006.05.002

10. A. Shirin and M. S. Islam, J. Sci. Res. 2 (2), 264 (2010). http://dx.doi.org/10.3329/jsr.v2i2.4483

11. P. E. Lewis and J. P. Ward, The Finite Element Method, Principles and Applications (AddisonWesley, Wokingham, England, 1991). 